Journal of Applied Fluid Mechanics, Vol. 9, Special Issue 1, pp. 165-171, 2016. Selected papers from the $7^{\text {th }}$ International Exergy, Energy

and Environment Symposium, IEEE7-2015

Available online at www.jafmonline.net, ISSN 1735-3572, EISSN 1735-3645.

DOI: $10.36884 /$ jafm.9.SI1.25817

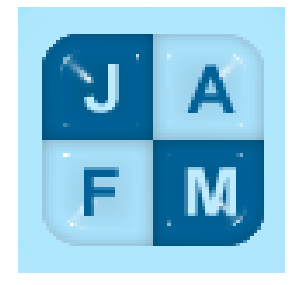

\title{
Effect of Dispersed Nanoparticles on Thermophysical Properties of Nanofluid and Heat Transfer Coefficients
}

\author{
H. Afshar ${ }^{1}$, M. Shams ${ }^{2}$, S. M. Mousavi Nainian ${ }^{3}$ and G. Ahmadi ${ }^{4}$ \\ ${ }^{1}$ Department of Mechanical Engineering, East Tehran Branch, Islamic Azad University, Tehran, Iran \\ ${ }^{2}$ Department of Mechanical Engineering, K. N. Toosi University of Technology, Tehran, Iran \\ ${ }^{3}$ Department of Mechanical Engineering, West Tehran Branch, Islamic Azad University, Tehran, Iran \\ ${ }^{4}$ Department of Mechanical and Aeronautical Engineering, Clarkson University, Potsdam NY, 13699, USA \\ †Corresponding AuthorEmail: Afshar@iauet.ac.ir
}

(Received October 20, 2015; accepted December 10, 2015)

\begin{abstract}
In this investigation, effects of dispersed $\mathrm{Cu}$ nanoparticles in water on heat transfer coefficients are studied using Eulerian-Lagranigian approach. Nanoparticles disperse in the fluid due to drag, weight and Brownian forces acting on them. A new particle search algorithm is used to trace the particles in every time step. Thermal coupling between dispersed and carrier phases is done and also thermal and momentum interaction between particles and solid walls are taken onto account to obtain velocity and temperature fields. The specific heat of nanofluid is obtained using conventional models. The results show that regarding thermophysical properties of particles and base fluid, and also other conditions like mass flow rate and particle size, degradation or intensification of heat transfer coefficients can occur.
\end{abstract}

Keywords: Nanofluid; Nanoparticle; Heat transfer; Two phase flow.

\section{NOMENCLATURE}

$\begin{array}{ll}\rho & \text { density } \\ k & \text { thermal conductivity } \\ \mathrm{Cp} & \text { specific heat } \\ \mu & \text { dynamic viscosity } \\ \mathrm{u}^{\mathrm{p}} & \text { nanoparticle velocity in the } \mathrm{x} \text { direction } \\ \mathrm{v}^{\mathrm{p}} & \text { nanoparticle velocity in the y direction }\end{array}$

\section{INTRODUCTION}

Particle-fluid two-phase flows are very common in both natural and industrial processes, which are characterized by nonlinear and non-equilibrium phenomena and involves multiscale processes. Significant work regarding nanoparticles and nanofluids thermo-physical properties began to surface mainly in the past decade; Hanley et al. (2012).Understanding the observed enhancement in heat transfer coefficient has involved many approaches ranging from existing theory alteration, by means of an empirical contribution, to numerical techniques, Yang et al. (2015).

Most findings have indicated a highly empirical nature and as a result, models based purely on theoretical means are very limited. Experimental results show that in natural convection, heat transfer coefficient decreases with increasing the

\author{
$\varphi \quad$ volume concentration of nanoparticles \\ $Q \quad$ heat transfer rate \\ $q$ heat flux convection coefficient \\ D thermal diffusivity \\ $\tau$ relaxation time
}

nanoparticle volume fraction, Nnanna (2007) and Chang (2008). Also there are experimental reports regarding the reduction of convective heat transfer coefficients in force convection (Kim S. et al. 2012).

Real mechanism of nanoscale heat transfer is not well understood yet, so the results of many investigations regarding heat transfer in nanofluids, does not match and even inconsistent, but many scientists, Evan (2006), compromise that Brownian motion of nanoparticles is the dominant mechanism of heat transfer in nanofluids. Decreasing the particle size to nano arena, this random motion influence becomes significant. Some propose Brownian motion is why larger sized particles quickly settle while smaller ones behave diffusively. It's believed this random motion increases energy transfer, through convection, between particle and fluid. This rests on the idea of 
spherical boundary layer formation around the particles. Xu et al. (2007) focused on multi scale modeling and studied the effect of meso-scale structures on the hydrodynamic behaviors of particle-fluid systems. They considered in an Eulerian-Lagrangian model utilizing the detailed particle distribution information. The fluid flow was distributed within each computational cell from pressure balance considerations according to weighted local porosities, rather than by using traditional linear interpolations. The drag on each particle was then calculated with aid of the local porosity and slip velocity. They claimed the approach showed significant differences to traditional method.

Considering a single nanoparticle in fluid flow, mean free path of fluid molecules will be comparable with particle diameter and rarefaction effects will play an important role in hydrodynamic and thermal interactions between particle and fluid. Fan and Wang (2010) theoretically examined the macroscale manifestation of the microscale physics in nanofluids. They claimed that the microscale interaction between nanoparticles and base fluids manifests itself as thermal waves/resonance at the macroscale. The energy equation that governs the heat transfer in nanofluids is of a dual-phase-lagging type instead of the postulated and commonly-used classical energy equation. The interplays among diffusion, convection, and thermal waves/resonance enrich the heat transfer in nanofluids considerably.

Many experimental and semi-experimental efforts are made regarding heat transfer in nanofluids but by the knowledge of the authors, no direct EulerianLagrangian heat transfer simulation is done. Afshar et al. (2009) studied microchannel heat transfer and dispersion of nanoparticles in slip flow regime with constant heat flux. They solved analytically the Navier-Stokes and energy equations for fluid flow in a microchannel in slip flow regime and discussed the effect of different parameters on heat transfer and dispersion of nanoparticles in microchannels.

A key element in any hybrid Eulerian-Lagrangian calculation procedure for two-phase flow is an algorithm by which particles are tracked in time and space within a fixed flow field. This algorithm involves the solution of well-established ODEs for particle motion and position along the particle's trajectory, with the instantaneous carrier velocity being extracted from the Navier-Stokes solution in conjunction with a dispersion model, in the case of Reynods Averaged Navier-Stokes (RANS) schemes. An important element of the algorithm is the determination of the particle's position within the discrete mesh of nodes at which properties of the continuous carrier phase are stored. The other important element is an effective interpolation of these properties to the particle's position, which is required to solve the equations governing the particle motion and thermal state.

In a finite volume scheme in which volume averaged mass, momentum and heat sources arising from inter-phase exchange must be determined, a supplementary task is to search for the finite volume within which the particle and the carrier exchange the relevant properties. Zhou and Leschziner (1999) proposed a scheme for tracking the position of particles within a fixed mesh used to compute the motion of a continuous carrier fluid. The principal output of this scheme was the identification of the grid cell within which the particle resides at any time during the tracking of its trajectory. This information is required for the solution of the particle equation of motion and for modeling the exchange processes between the particles and surrounding fluid. Afshar et al. (2010) introduced LNR search algorithm as an efficient algorithm for nanofluid applications.

In this study, using binary tree and the left-noderight (LNR) algorithm which is very efficient for complex geometries or unstructured grids, the dispersion of nanoparticles in fluid flow and the resulting three-way heat transfer are analyzed. The applications to heat conducting nanfluids and related heat transfer coefficients are also discussed.

\section{PROBLEM DEFINITION}

The geometry of the present study consists of a twodimensional rectangular duct of height $2 \mathrm{H}$ and length L (Fig 1). Top and bottom walls of the channel are set to a constant temperature of $30^{\circ} \mathrm{C}$. Cold nanofluid of temperature $20^{\circ} \mathrm{C}$ enters with a uniform velocity of $0.5 \mathrm{~m} / \mathrm{s}$ to the channel and flows from left to right. As the fluid flows through the duct, it takes away heat from the top and bottom walls.

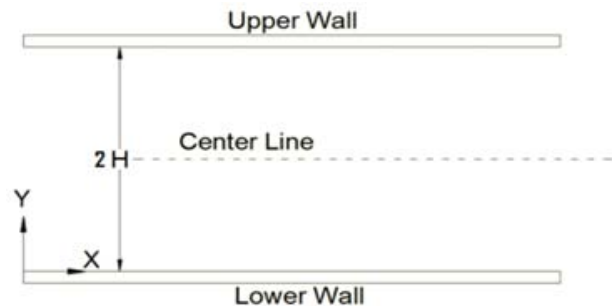

Fig. 1. Channel Geometry.

Channel height is 100 micrometers and its length is 1 centimeter. The nanofluid is a mixture of water and solid spherical copper particles of $100 \mathrm{~nm}$ diameter which are of uniform in shape and size. The nanofluid is considered to be incompressible and the flow is laminar. The thermophysical properties of the nanofluid are assumed to be constant. Effect of drag, gravity and Brownian forces on acceleration of each nanoparticle is taken into account. Thermophysical properties of water and copper are listed in table 1 .

Table 1 Thermophysical properties of water and copper

\begin{tabular}{|c|c|c|}
\hline Property & Water & Copper \\
\hline$\rho\left(\mathrm{kg} / \mathrm{m}^{3}\right)$ & 1000.52 & 8954.0 \\
\hline $\mathrm{C}_{\mathrm{p}}(\mathrm{j} / \mathrm{kg} . \mathrm{K})$ & 4181.8 & 383.1 \\
\hline $\mathrm{k}(\mathrm{W} / \mathrm{m} . \mathrm{K})$ & 0.597 & 386.0 \\
\hline$\mu(\mathrm{Pa} . \mathrm{s})$ & $1.002 \times 10^{-3}$ & ------- \\
\hline
\end{tabular}




\section{GOVERNING EQUATIONS}

\subsection{Carrier phase}

In two dimensional incompressible fluid flow, the governing equations are given as:

Continuity

$$
\frac{\partial u}{\partial x}+\frac{\partial v}{\partial y}=0
$$

Momentum

$u \frac{\partial u}{\partial x}+v \frac{\partial u}{\partial y}=-\frac{1}{\rho} \frac{\partial p}{\partial x}+\frac{\mu}{\rho}\left(\frac{\partial^{2} u}{\partial x^{2}}+\frac{\partial^{2} u}{\partial y^{2}}\right)$

$u \frac{\partial v}{\partial x}+v \frac{\partial v}{\partial y}=-\frac{1}{\rho} \frac{\partial p}{\partial y}+\frac{\mu}{\rho}\left(\frac{\partial^{2} v}{\partial x^{2}}+\frac{\partial^{2} v}{\partial y^{2}}\right)$

Energy

$$
\rho c_{p}\left(u \frac{\partial T}{\partial x}+v \frac{\partial T}{\partial y}\right)=k\left(\frac{\partial^{2} T}{\partial x^{2}}+\frac{\partial^{2} T}{\partial y^{2}}\right)
$$

\subsection{Discrete phase}

Particles move in $\mathrm{x}$ and $\mathrm{y}$ directions due to drag, weight and Brownian forces. That is

$$
\begin{aligned}
& \frac{d u^{p}}{d t}=\frac{1}{\tau}\left(u-u^{p}\right)+n_{1}(t) \\
& \frac{d v^{p}}{d t}=\frac{1}{\tau}\left(v-v^{p}\right)+n_{2}(t)-g \\
& \tau=\frac{\rho^{p} C_{c} d^{2}}{18 \mu}
\end{aligned}
$$

Here $\mathrm{n} 1(\mathrm{t})$ and $\mathrm{n} 2(\mathrm{t})$ are the Brownian random forces that are evaluated at each time step using the procedure described by Li and Ahmadi (1992). In Equations (5) and (6), up and vp stand for particle velocity in $\mathrm{x}$ and $\mathrm{y}$ directions. $\mathrm{Cc}$ is Cunningham correction factor, $\mathrm{d}$ is particle diameter and $\tau$ is relaxation time of nanoparticles. Superscript $p$ refers to particle property.

Particle diameter is in nanometer range, so we conclude that according to nanolayering effects and phonon heat transfer mechanism according to Chong et al. (2004) and Avsec (2008); at every time step it is locally in thermal equilibrium with the surrounding medium.

Each particle transfers energy as it moves from a control volume to another control volume as shown schematically in Fig 2. As noted before, the motion of the particle occurs because of Brownian motion, drag or weight forces. Temperature variation in node points of continuous phase due to energy transferred by the nanoparticles is approximated (Hou and Komanduri (2000)) using
$\Delta \mathrm{T}=\frac{\mathrm{q}}{4 \mathrm{k} \pi}\left[-0.5772+\ln \left(\frac{4 \mathrm{Dt}}{\mathrm{r}^{2}}\right)\right]$

Where $\mathrm{q}$ is the heat flux transferred by nanoparticles, $\mathrm{k}$ is the heat conductivity of fluid, $\mathrm{r}$ is the distance between the nanoparticle and computational nodes, $\mathrm{D}$ is thermal diffusivity, $\mathrm{t}$ is time and 0.5772 is the Euler's constant.

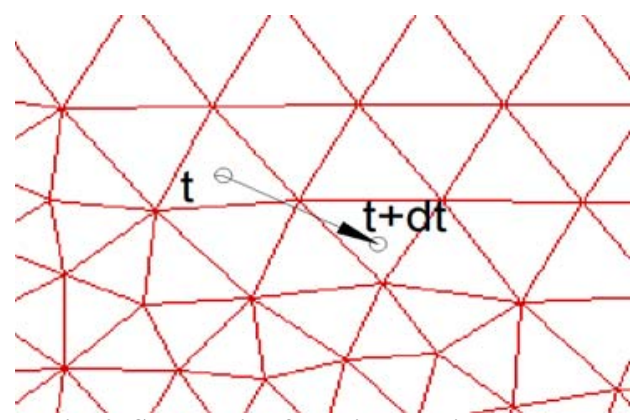

Fig. 2. Schematic of particle motion between control volumes in every time steps.

\section{NUMERICAL SIMULATION}

The governing mass, momentum and energy equations for the carrier phase has been discretized by a control volume approach using a power law profile approximation. The set of discretized equations have been solved iteratively using the SIMPLE algorithm.

After obtaining velocity and temperature fields of the base fluid, copper nanoparticles of $100 \mathrm{~nm}$ in size are dispersed with different volume fraction concentration to investigate the effects of thermophysical property variation of the resultant nanofluid on the prediction of the convective heat transfer coefficient. The heat transfer coefficient of flowing nanofluid can be calculated from following equations.

The heat that is carried by the fluid (water or nanofluid) is defined as:

$Q=\dot{m} c_{p}\left(T_{\text {in }}-T_{\text {out }}\right)$

Where $\mathrm{Q}$ is the heat transfer rate of the fluid and $\mathrm{m}$ is the mass flow rate of the fluid. Convection heat transfer coefficient can be calculated by its definition:

$h=\frac{Q}{A\left(T_{s}-T\right)}$

A is the microchannel pyramid, Ts is the wall temperature, Tin and Tout are the average fluid temperature in the entrance and exit of the microchannel and $\mathrm{T}$ is the average fluid temperature in each cross section As that is calculated as follows: 
H. Afshar et al. /JAFM, Vol. 9, Special Issue 1, pp. 165-171, 2016.

$T_{\text {average }}=\frac{\int u T d A_{s}}{A_{s} u_{m}}$

This carried heat enters into the microchannel by heat conduction mechanism through its walls:

$Q=-\left.k A \frac{d T}{d y}\right|_{w}$

$\mathrm{k}$ is the fluid thermal conductivity.

In the case of nanofluid, there are only two wellknown models for calculation of the specific heat capacity at constant pressure. Model I is according to volumetric averaging of specific heat capacity:

$C_{p, \text { nanofluid }}=\varphi C_{p, C u}+(1-\varphi) C_{p, \text { water }}$

Model II is volumetric averaging between volumetric heat capacities of base fluid and nanoparticle:

$C_{p, \text { nanofluid }}=\frac{\varphi\left(\rho C_{p}\right)_{c u}+(1-\varphi)\left(\rho C_{p}\right)_{\text {water }}}{\varphi \rho_{C u}+(1-\varphi) \rho_{\text {water }}}$

Where $\Phi$ is the volume concentration of nanoparticle.

Both models are often cited by a number of researchers for calculating the specific heat of nanofluid. However, the first model is approximately correct only for dilute suspensions when small density difference exists between base fluid and nanoparticle. For water-Cu nanofluid, the deviation of first model cannot be ignored, as the density ratio between nanoparticle and base fluid is large $(\rho c u=8954 \mathrm{~kg} / \mathrm{m} 3$, while $\rho$ water $=1000.52$ $\mathrm{kg} / \mathrm{m} 3$ ), so that large discrepancy occurs when increasing the volume concentration of nanoparticle. Details of these two models are discussed by Santra (2009) and Hanley et al. (2012)

Through numerical procedure for every nanoparticle concentration, mean temperatures and wall temperature gradient in the nanofluid will be calculated and so thermal conductivity and convection coefficient of nanofluid can be calculated.

\subsection{Code validation}

For water flow in duct, the fully developed length has beencalculated and compared with the results of Santraet al. (2009) and correlation given byDurst et al. (2005). The results are summarized in Table (2). It has been observed that the calculated entrance length by the present code is in good agreement by the results of Durst and Santra. For Eulerian-Lagrangian approach in modeling nanofluids, no study through two-dimensional microchannel has been found to validate the code.
Table 2 Entrance length (\%) for water flow in the microchannel for different Reynolds numbers

\begin{tabular}{|c|c|c|c|}
\hline Re & Durst et al. & Santraet al. & $\begin{array}{c}\text { Present } \\
\text { study }\end{array}$ \\
\hline 10 & 0.835087 & 0.813569 & 0.859 \\
\hline 20 & 1.177987 & 1.204162 & 1.105 \\
\hline 50 & 2.391482 & 2.569990 & 2.463 \\
\hline 100 & 4.541651 & 4.927244 & 4.683 \\
\hline
\end{tabular}

\section{RESULTS}

Temperature and velocity counters for Reynolds numbers of 100 and 10 are shown in Figs 3 and 4 respectively. It can be observed that hydrodynamic fully developed length is very small in comparison to thermal development length for both cases.

For Reynolds number of 10, thermal development length is less than half of the channel and for Reynolds number of 100; the flow will not reach a thermal developing condition. Channel walls are in constant temperature so in thermal fully developed region, temperature will be constant. As mentioned, Borwnian motion of nanoparticles is the dominant mechanism of energy transfer in nanofluids. For a constant temperature no energy will be transferred due to particle migration and naoparticles will not affect the
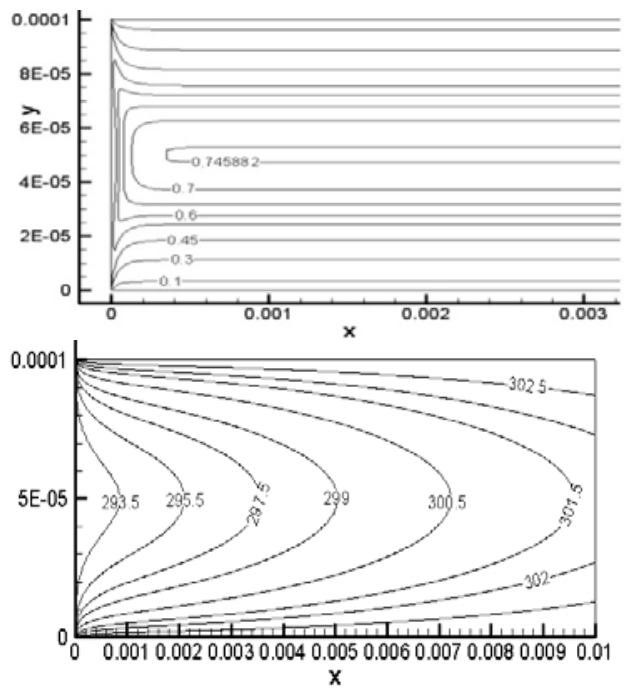

Fig. 3. $R e=100$, (a) Velocity counters, (b) Temperature counters.

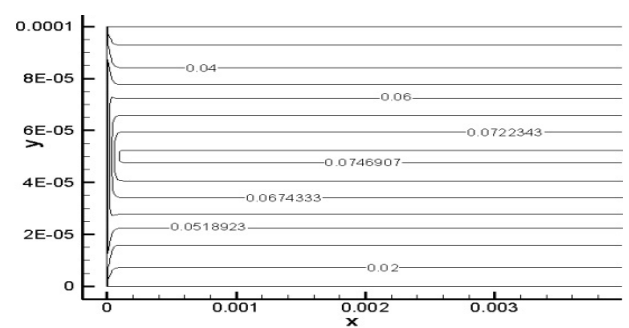




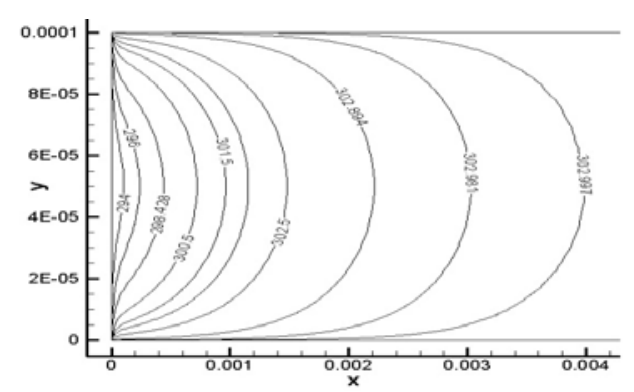

Fig. 4. $R e=10$, (a) Velocity counters, (b) Temperature counters.

temperature field. So it can be concluded that in the present configuration, nanofluids would not be applicable for Reynolds numbers less than 100 .

Effect of nanoparticle volume fraction on thermal coefficients for $\mathrm{Re}=100$ will be discussed.

Using equations (13) and (14), specific heat capacity variation of nanofluid versus volume fraction is shown in Fig 5.

Even for low volume fractions of $\mathrm{Cu}$ nanoparticles, specific heat capacity reduces considerably. Specific heat capacity of nanofluid predicted by equation (14) is less than that predicted by equation (13). But because of high relative density of $\mathrm{Cu}$ nanoparticles in comparison to water, equation (14) is more accurate for this study.

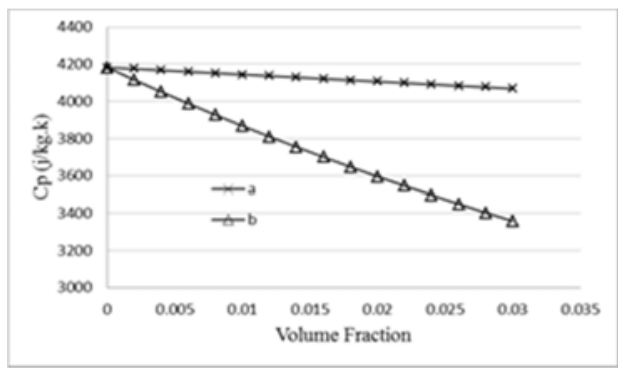

Fig. 5.Variation of specific heat capacity versus.

volume fraction of nanoparticles. (a) Equation (13), (b) Equation (14)

Using equation (9) and concerning the effect of nanoparticles on thermophysical properties, heat transfer for different volume fractions is summarized in table 3 .

Table 3 Variation of heat transfer due to reduced specific heat capacity of nanofluid

\begin{tabular}{|c|c|c|c|c|}
\hline $\begin{array}{c}\text { Volume } \\
\text { Fraction }(\varnothing)\end{array}$ & 0 & 0.005 & 0.01 & 0.02 \\
\hline $\begin{array}{c}\mathrm{Q} / \text { Qbase } \\
\text { fluid }(\%)\end{array}$ & 100 & $\begin{array}{c}99.90 \\
992\end{array}$ & $\begin{array}{c}99.819 \\
87\end{array}$ & $\begin{array}{c}99.639 \\
73\end{array}$ \\
\hline
\end{tabular}

Addition of $\mathrm{Cu}$ nanoparticles to fluid flow results an increment in mass flow rate, also it causes reduction of specific heat capacity. The net effect for this configuration is reduction in heat transfer rate.

Calculating the temperature field in nanofluid during numerical procedure, temperature gradients near the walls will be obtained. Convective heat transfer rate is equal to conductive heat transfer rate in walls of the microchannel. Variation of thermal conductivity versus nanoparticle volume fraction is summarized in table 4 .

Table 4 Variation of thermal conductivity versus $\mathrm{Cu}$ nanoaprticle volume fraction

\begin{tabular}{|c|c|c|c|c|}
\hline $\begin{array}{c}\text { Volume } \\
\text { Fraction }(\varnothing)\end{array}$ & 0 & 0.005 & 0.01 & 0.02 \\
\hline $\begin{array}{c}\mathrm{k} / \mathrm{kbase} \\
\text { fluid (\%) }\end{array}$ & 100 & $\begin{array}{c}99.910 \\
07\end{array}$ & $\begin{array}{c}99.820 \\
33\end{array}$ & $\begin{array}{c}99.646 \\
57\end{array}$ \\
\hline
\end{tabular}

It shows that by increasing the volume fraction, effective thermal conductivity of nanofluid reduces. If nanoparticles affected the temperature field near the wall in such a way that decreases the temperature gradients, it would cause increasing in the effective thermal conductivity.

Fig 6 shows the displacement of nanoparticles in the microchannel. Nanoparticles follow the streamlines but because of Brownian motion, trajectories are not symmetric. Energy transfer by the mass of particles occurs in every direction due to Brownian motion of nanoparticles.

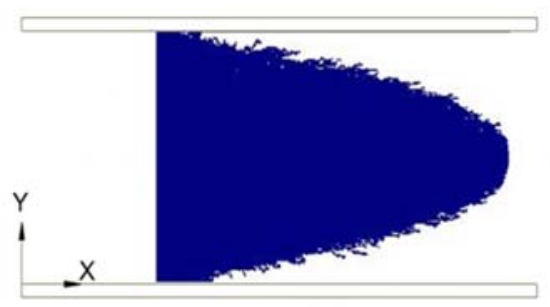

Fig. 6. Dispersion of nanoparticles in the microchannel.

Effect of $2 \%$ volume fraction of nanoparticles on temperature filed is shown in Fig 7. It shows that temperature gradients near the wall are slightly increased which caused a reduction in thermal conductivity.

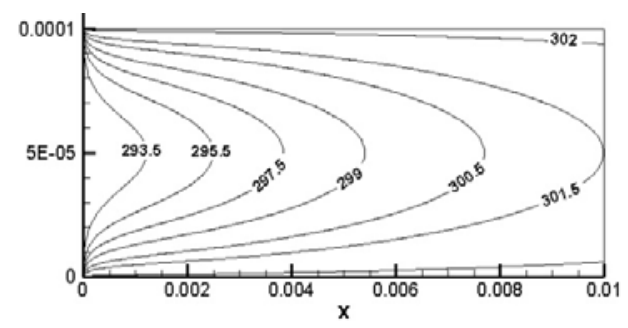

Fig. 7. Temperature counters for volume fraction of $2 \%$.

Variation of convective heat transfer coefficient along the microchannel for different volume fractions of nanoparticles is shown in Figs 8, 9 and 
10.

It shows that increasing the concentration of nanoparticles causes a reduction in convective heat transfer coefficient. The difference between this coefficient for base fluid and resultant nanofluid is less in the entrance of the microchannel but this difference increases along the microchannel to reach a constant value.

\section{CONCLUSION}

Dispersion of $\mathrm{Cu}$ nanoparticles with $100 \mathrm{~nm}$ diameter in water with different volume is studied numerically. Nanoparticles disperse randomly in the

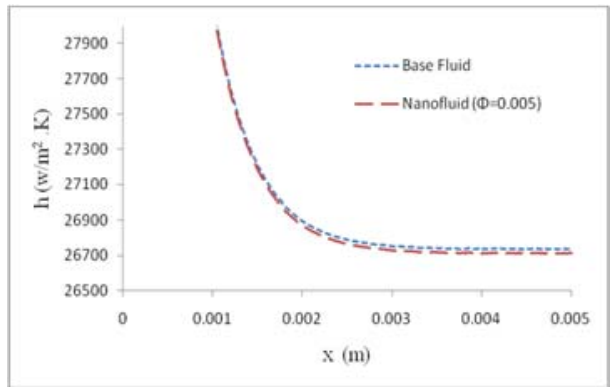

Fig. 8. Variation of convective heat transfer coefficient for solid volume fraction of $\mathbf{0 . 0 0 5}$ $(\mathbf{R e}=100)$.

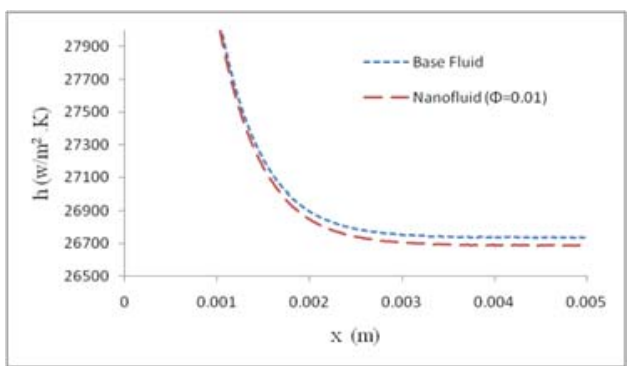

Fig. 9. Variation of convective heat transfer coefficient for solid volume fraction of $\mathbf{0 . 0 1}$ $(\mathrm{Re}=100)$.

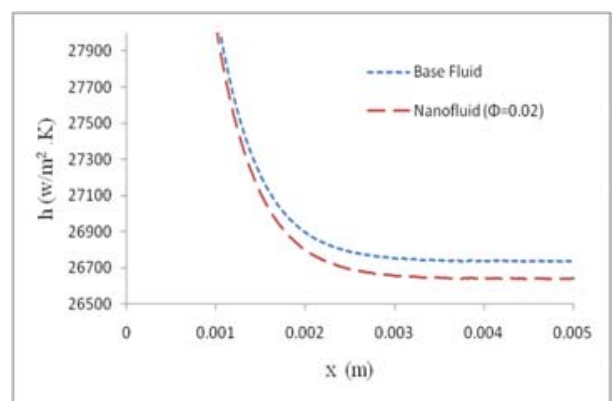

Fig. 10. Variation of convective heat transfer coefficient for solid volume fraction of $\mathbf{0 . 0 2}$ $(\mathrm{Re}=100)$.

fluid because of drag, weight and Brownian forces and carry energy in every direction. But the average displacement is in such a way that they follow the stream lines.

As there are inconsistent reports about heat transfer in nanofluids, so well known relations are used to calculate thermophysical properties and numerical procedure is employed for calculation of heat transfer coefficients. Density of $\mathrm{Cu}$ is very higher than the density of water so volumetric averaging between volumetric heat capacities of base fluid and nanoparticle is used to estimate the heat capacity of nanofluid. Specific heat of $\mathrm{Cu}$ nanoparticles is very lower than water specific heat. Even for low volume fraction, the specific heat capacity of nanofluid reduces considerably in comparison to base fluid. Density of $\mathrm{Cu}$ nanoparticles is very higher than water density and after injection of nanoparticles, the mass flow rate increases. Heat transfer in nanofluid can be reduced or increased regarding the balance between the increased mass flow rate and decreased specific heat.

Energy transfer by the mass of nanoparticles, affect the temperature field and temperature gradients near the walls. Balance of conductive and convective heat transfer in the walls can lead to obtain the effective thermal conductivity of nanofluid.

Cpu time for $2 \%$ volume fraction of nanoparticles is about 100 hours using a $3.2 \mathrm{GHz}$ processor.

\section{REFERENCES}

Afshar, H., M. Shams, S.M.M. Nainian, and G. Ahmadi (2009). Microchannel heat transfer and dispersion of nanoparticles in slip flow regime with constant heat flux. International Communications in Heat and Mass Transfer, 36, 1060-1066.

Afshar, H. M. Shams, S. M. M. Nainian, and G. Ahmadi (2010, July) .A search algorithm for particle laden flows: application to nanofluids. Proceedings of ASME Biennial Conference on Engineering Systems Design and Analysis, Istanbul, Turkey.

Avsec, J. (2008). The combined analysis of phonon and electron heat transfer mechanism on thermal conductivity for nanofluids, International Journal of Heat and Mass Transfer 51, 45894598 .

Chang, B. H., A. F. Mill, and E. Hernandez (2008). Natural convection of microparticle suspensions in thin enclosures, International Journal of Heat and Mass Transfer, 51, 1332-1341.

Chung J. D., A. J. H. Mc Gaughey and M. Kaviany (2004, June).Role of Phonon Dispersion in Lattice Thermal Conductivity Modeling.Transactions of the ASME, 126.

Durst F., S. Ray, B. Unsal, and O. A. Bayoumi (2005). The development lengths of laminar pipe and channel flows. ASME J. Fluids 
H. Afshar et al. /JAFM, Vol. 9, Special Issue 1, pp. 165-171, 2016.

Engng., 127, 1154-1160.

Evans W., J. Fish, and P. Keblinski (2006). Role of Brownian motion hydrodynamics on nanofluid thermal conductivity, Applied physics letters, 88, 093116.

Fan J. and L. Wang (2010). Is Classical Energy Equation Adequate for Convective Heat Transfer in Nanofluids?. Advances in Mechanical Engineering.

Hou Z. B. and R. Komanduri (2000). General solutions for stationary/moving plane heat source problems in manufacturing and tribology. International Journal of Heat and Mass Transfer, 43, 1679-1698.

Kim S., H. Yoo, and C. Kim (2012).Convective heat transfer of alumina nanofluids in laminar flows through a pipe at the thermal entrance regime, Korean Journal of Chemical Engineering, (29) 10,1321-1328.

Li A., and G. Ahmadi (1992). Dispersion and Deposition of Spherical Particles from Point Sources in a Turbulent Channel Flow, Aerosol Science and Technology.

Nnanna A. G. (2007). Experimental model of temperature-driven nanofluid. ASME Transactions, Journal of Heat Transfer, 129, 697-704.

O’Hanley H., J. Buongiorno, T. McKrell, and L.
$\mathrm{Hu}(2012)$.Measurement and Model Validation of Nanofluid Specific Heat Capacity with Differential Scanning Calorimetry.Hindawi Publishing Corporation. Advances in Mechanical Engineering, Article ID 181079.

Prasher, R., P. Bhattacharya, andP.E. Phelan(2005).Thermal conductivity of nanoscale colloidal solutions (nanofluids). Physical Review Letters, 94 (025901), 1-4.

Santra A. K., S. Sen, andN. Chakraborty (2009). Study of heat transfer due to laminar flow of copper-water nanofluid through two isothermally heated parallel plates. International Journal of Thermal Sciences, 48, 391-400.

Xu M., W. Ge, andJ. Lia (2007). A discrete particle model for particle-fluid flow with considerations of sub-grid structures, Chemical Engineering Science, 62,2302-2308.

Yang J. C., F. C. Li, H. P. Xu, Y. R He, Y. M. Huang and B. C. Jiang (2015). Heat Transfer Performance Of Viscoelastic-Fluid-Based Nanofluid Pipe Flow At Entrance Region, Experimental Heat Transfer, (28) 2.

Zhou Q. and M. A. Leschziner (1999). An improved particle-locating algorithm for Eulerian-Lagrangian computations of two-phase flows in general Coordinates. International Journal of Multiphase Flow, 25, 813-825. 